\title{
A STRUCTURE THEOREM FOR DIFFERENTIAL ALGEBRAS
}

\author{
MARCUS TRESSL \\ NWF-I Mathematik, Universität Regensburg, 93040 Regensburg, Germany \\ E-mail: marcus.tressl@mathematik.uni-regensburg.de
}

The theorem mentioned in the title is

TheOREM 1. Let $S=\left(S, \partial_{1}, \ldots, \partial_{K}\right)$ be a differential domain in $K$ commuting derivatives, containing $\mathbb{Z}$ and let $R=\left(R, \partial_{1}, \ldots, \partial_{K}\right) \subseteq\left(S, \partial_{1}, \ldots, \partial_{K}\right)$ be a differential subring such that $S$ is differentially finitely generated over $R$. Then there are $R$-subalgebras $B$ and $P$ of $S$ and an element $h \in B, h \neq 0$ such that:

(a) $B$ is a finitely generated $R$-algebra and $B_{h}$ is a finitely presented $R$-algebra.

(b) $S_{h}=(B \cdot P)_{h}$ is a differentially finitely presented $R$-algebra.

(c) The homomorphism $B \otimes_{R} P \rightarrow B \cdot P$ induced by multiplication is an isomorphism of $R$-algebras.

(d) $P$ has the following structure. For each subset $\Delta$ of $\left\{\partial_{1}, \ldots, \partial_{K}\right\}$ there is an $R$ subalgebra $P_{\Delta}$ of $P$ such that $P_{\Delta}$ together with the derivatives from $\Delta$ is a differential polynomial ring in these derivatives and finitely many variables (the case $P_{\Delta}=R$ is not excluded). The homomorphism

$$
\bigotimes_{\Delta \subseteq\left\{\partial_{1}, \ldots, \partial_{K}\right\}} P_{\Delta} \rightarrow P
$$

induced by multiplication is an isomorphism of R-algebras.

If $R$ is a differential ring as in the theorem, then a differential $R$-algebra $S$ is a quotient of a differential polynomial ring $R\{Y\}$ over $R$ modulo a differential ideal $\mathfrak{a}$. One of the fundamental tools of differential algebra is a reduction process of polynomials $F \in R\{Y\}$ with respect to such ideals as explained in Kolchin's book [2]; provided $\mathbb{Z} \subseteq R \subseteq S$ and $S$ is a domain. We translate the result of this reduction in terms of the differential algebra $S$. In the case where $R=\mathbb{R}$ is the field of real numbers and the number of derivatives $K$ is 1 , our structure theorem can be used to reduce the solvability of an ordinary system of differential equations to an algebraic question on the system. This is done in [1].

2000 Mathematics Subject Classification: Primary 12H05; Secondary 13N.

The paper is in final form and no version of it will be published elsewhere. 
In section 1 we recall the definition of a characteristic set (in characteristic 0 ) from [2]. In section 2 we recall the result of the reduction process with respect to characteristic sets and how differential prime ideals can be recovered from their characteristic sets. In section 3 we translate these facts into the proof of Theorem 1.

1. Definition of characteristic sets. Let $R$ be a differential ring in $K$ pairwise commuting derivatives $\partial_{1}, \ldots, \partial_{K}$. Let $Y:=\left(Y_{1}, \ldots, Y_{N}\right)$ be a tuple of $N$ indeterminates over $R$ and let $\mathcal{D}:=\left\{\partial_{1}^{i_{1}} \ldots \partial_{K}^{i_{K}} \mid i_{1}, \ldots, i_{K} \in \mathbb{N}_{0}\right\}$ be the free abelian monoid generated by $\left\{\partial_{1}, \ldots, \partial_{K}\right\}$, which we denote multiplicatively. For each $D \in \mathcal{D}$ and $n \in\{1, \ldots, N\}$ let $D Y_{n}$ be an indeterminate, where $D Y_{n}=Y_{n}$ if $D=\partial_{1}^{0} \ldots \partial_{K}^{0}$ by definition. Moreover let

$$
\mathcal{D} Y:=\left\{D Y_{n} \mid D \in \mathcal{D}, 1 \leq n \leq N\right\} .
$$

The differential polynomial ring over $R$ in $K$ derivatives and $N$ indeterminates is the polynomial ring $R\{Y\}:=R[y \mid y \in \mathcal{D} Y]$ together with the uniquely determined derivations $\partial_{i}$ such that $\partial_{i}\left(r \cdot D Y_{n}\right)=\left(\partial_{i} r\right) \cdot D Y_{n}+r \cdot\left(\partial_{i} D\right) Y_{n}(1 \leq i \leq K, 1 \leq n \leq N, r \in R)$. So $R\{Y\}$ is a differential ring extension of $R$ and $R\{Y\}$ is the free object generated by $N$ elements over $R$ in the category of differential rings with $K$ commuting derivatives. The set of all powers of variables from $\mathcal{D} Y$ is denoted by

$$
\mathcal{D} Y^{*}:=\left\{y^{p} \mid y \in \mathcal{D} Y, p \in \mathbb{N}\right\} .
$$

Definition 1. The rank on $\mathcal{D} Y^{*}$ is the map rk: $\mathcal{D} Y^{*} \rightarrow \mathbb{N}_{0} \times\{1, \ldots, N\} \times \mathbb{N}_{0}^{K} \times \mathbb{N}$ defined by

$$
\operatorname{rk}\left(\partial_{1}^{i_{1}} \ldots \partial_{K}^{i_{K}} Y_{n}\right)^{p}:=\left(i_{1}+\ldots+i_{K}, n, i_{K}, \ldots, i_{1}, p\right) .
$$

The set $\mathcal{O}:=\mathbb{N}_{0} \times\{1, \ldots, N\} \times \mathbb{N}_{0}^{K} \times \mathbb{N}$ equipped with the lexicographic order (hence the first component is the dominating one) is well ordered. Note that the order type of the image of rk in $\mathcal{O}$ is the order type of $\mathbb{N}$.

Definition 2. We say a variable $y \in \mathcal{D} Y$ appears in $f \in R\{Y\}$ if $y$ appears in $f$ considered as an ordinary polynomial (hence $Y_{1}$ does not appear in $\partial_{1} Y_{1}$ ). The leader $u_{f}$ of $f \in R\{Y\} \backslash R$ is the variable $y \in \mathcal{D} Y$ of highest rank which appears in $f$. Moreover $u_{f}^{*}:=u_{f}^{\operatorname{deg}_{u_{f}} f} \in \mathcal{D} Y^{*}$ denotes the highest power of $u_{f}$ in $f$. We extend the rank to polynomials $f \in R\{Y\}$ by

$$
\operatorname{rk}(f):=\operatorname{rk}\left(u_{f}^{*}\right) \in \mathcal{O} .
$$

Definition 3. If $g, f \in R\{Y\}, g \notin R$ are polynomials, then $f$ is called weakly reduced with respect to $g$ if no proper derivative of $u_{g}$ appears in $f . f$ is called reduced with respect to $g$ if $f$ is weakly reduced with respect to $g$ and if $\operatorname{deg}_{u_{g}} f<\operatorname{deg}_{u_{g}} g$.

The polynomial $f$ is called (weakly) reduced with respect to a nonempty set $G \subseteq$ $R\{Y\} \backslash R$ if $f$ is (weakly) reduced with respect to every $g \in G$.

A nonempty subset $G \subseteq R\{Y\} \backslash R$ is called autoreduced if every $f \in G$ is reduced with respect to all $g \in G, g \neq f$. If $G$ consists of a single element then $G$ is called autoreduced as well.

It is easy to see that $u_{f} \neq u_{g}$ (hence $\mathrm{rk} f \neq \mathrm{rk} g$ ) if $f, g$ are different polynomials from an autoreduced set. Moreover, by [2], Chap. O, Section 17, Lemma 15 (a) we have 
Proposition 2. Every autoreduced set is finite.

Let $\infty$ be an element bigger than every element in $\mathcal{O}$ and let $(\mathcal{O} \cup\{\infty\})^{\mathbb{N}}$ be equipped with the lexicographic order. We define the rank of an autoreduced set $G$ to be an element of $(\mathcal{O} \cup\{\infty\})^{\mathbb{N}}$ as follows. Let $G=\left\{g_{1}, \ldots, g_{l}\right\}$ with rk $g_{1}<\ldots<\operatorname{rk} g_{l}$. Then

$$
\operatorname{rk} G:=\left(\operatorname{rkg} g_{1}, \ldots, \operatorname{rk} g_{l}, \infty, \infty, \ldots\right) .
$$

Proposition 3. There is no infinite sequence $G_{1}, G_{2}, \cdots$ of autoreduced sets with the property $\operatorname{rk} G_{1}>\operatorname{rk} G_{2}>\cdots$.

Proof. [2], Chap. I, Section 10, Proposition 3.

Definition 4. If $M \subseteq R\{Y\}$ is a set not contained in $R$, then by Proposition 3 the set $\{\operatorname{rk} G \mid G \subseteq M$ is autoreduced $\}$ has a minimum. Every autoreduced subset $G$ of $M$ with this rank is called a characteristic set of $M$.

Proposition 4. If $G$ is a characteristic set of $M \subseteq R\{Y\}$ and $f \in M \backslash R$, then $f$ is not reduced with respect to $G$.

Proof. If $f \in M \backslash R$ is reduced with respect to $G$, then the set $\{g \in G \mid \operatorname{rk} g<$ $\operatorname{rk} f\} \cup\{f\}$ is an autoreduced subset of $M$ of rank strictly lower than the rank of $G$, which is impossible.

2. Fundamental properties of characteristic sets. From now on we assume that $R$ is a differential domain in $K$ derivatives containing $\mathbb{Z}$.

Definition 5. Let $f \in R\{Y\} \backslash R, f=f_{d} u_{f}^{d}+\ldots+f_{1} u_{f}+f_{0}$ with polynomials $f_{d}, \ldots, f_{0} \in R\left[y \in \mathcal{D} \mid y \neq u_{f}\right]$ and $f_{d} \neq 0$. The initial $I(f)$ of $f$ is defined as

$$
I(f):=f_{d} .
$$

The separant $S(f)$ of $f$ is defined as

$$
S(f):=\frac{d}{d u_{f}} f=d \cdot f_{d} u_{f}^{d-1}+\ldots+f_{1} .
$$

Moreover, for every autoreduced subset $G=\left\{g_{1}, \ldots, g_{l}\right\}$ of $R\{Y\}$ we define

$$
H(G):=\prod_{i=1}^{l} I\left(g_{i}\right) \cdot S\left(g_{i}\right) \text { and } H_{G}:=\left\{\prod_{i=1}^{l} I\left(g_{i}\right)^{n_{i}} S\left(g_{i}\right)^{m_{i}} \mid n_{i}, m_{i} \in \mathbb{N}_{0}\right\} .
$$

Since $R$ is a domain and $\mathbb{Z} \subseteq R$ the set $H_{G}$ does not contain 0. Moreover, $S(g)$ and $I(g)$ are reduced with respect to $G(g \in G)$.

TheOrem 5. Let $G \subseteq R\{Y\}$ be an autoreduced set and let $f \in R\{Y\}$. Let $[G]$ denote the differential ideal generated by $G$ in $R\{Y\}$ and let $(G)$ denote the ideal generated by $G$ in $R\{Y\}$. Then there is some $\tilde{f} \in R\{Y\}$ which is reduced with respect to $G$ and some $H \in H_{G}$ such that $H \cdot f \equiv \tilde{f} \bmod [G]$. If $f$ is weakly reduced with respect to $G$, then we can take $H$ such that $H \cdot f \equiv \tilde{f} \bmod (G)$.

Proof. [2], Chap. I, Section 9, Proposition 1. 
COROllary 6. If $G$ is a characteristic set of a differential prime ideal $\mathfrak{p}$ of $R\{Y\}$ with $\mathfrak{p} \cap R=0$ then

$$
\mathfrak{p}=\left\{f \in R\{Y\} \mid H(G)^{n} \cdot f \in[G] \text { for some } n \in \mathbb{N}_{0}\right\} .
$$

Moreover if $f \in \mathfrak{p}$ is weakly reduced with respect to $G$, then $H(G)^{n} \cdot f \in(G)$ for some $n \in \mathbb{N}_{0}$.

Proof. From Theorem 5 and Proposition 4, since $H_{G} \cap \mathfrak{p}=\emptyset$.

3. Proof of Theorem 1. Since $S$ is a differentially finitely generated $R$-algebra, there is some $N \in \mathbb{N}$ and a surjective differential homomorphism $\varphi: R\left\{Y_{1}, \ldots, Y_{N}\right\} \rightarrow S$. Let $Y:=\left(Y_{1}, \ldots, Y_{N}\right)$ and let $\mathfrak{p}$ be the kernel of $\varphi$. Since $R \subseteq S$ and $S$ is a differential domain, the ideal $\mathfrak{p}$ is a differential prime ideal of $R\{Y\}$ with $\mathfrak{p} \cap R=0$. Let $G$ be a characteristic set of $\mathfrak{p}$ (c.f. Definition 4). First we define $B, P$ and $h$. We take $h:=\varphi(H(G))(H(G)$ is defined in Definition 5),

$$
\begin{gathered}
V:=\left\{y \in \mathcal{D} Y \mid y \text { is not a proper derivative of any } u_{g}\right\}, \\
V_{B}:=\{y \in V \mid y \text { appears in some } g \in G\}, \\
B:=\varphi\left(R\left[V_{B}\right]\right) \text { and } P:=\varphi\left(R\left[V \backslash V_{B}\right]\right) .
\end{gathered}
$$

Since $G$ is an autoreduced set, a polynomial $f \in R\{Y\}$ is weakly reduced with respect to $G$ if and only if $f \in R[V]$.

Claim 1. The restriction of $\varphi$ to the subring $R\left[V \backslash V_{B}\right]$ of $R\{Y\}$ is injective.

Proof. Let $f \in R\left[V \backslash V_{B}\right] \cap \mathfrak{p}$. Since $f$ is weakly reduced with respect to $G$ and all leaders of elements $g \in G$ are in $V_{B}$ we have that $f$ is reduced with respect to $G$. Since $G$ is a characteristic set of $\mathfrak{p}$ we get $f=0$ from Proposition 4 and $\mathfrak{p} \cap R=0$. This proves the claim.

Claim 2. $h \neq 0$ and $S_{h}=(B \cdot P)_{h}$.

Proof. Since every $S(g), I(g)$ with $g \in G$ is reduced with respect to $G$ we have $H(G) \notin$ p. As $H(G) \in R\left[V_{B}\right]$ it follows $B \ni h=\varphi(H(G)) \neq 0$.

Let $f \in R\{Y\}$. By Theorem 5 there is some $\tilde{f} \in R\{Y\}$ which is reduced with respect to $G$ and some $H \in H_{G}$ such that $H \cdot f \equiv \tilde{f} \bmod [G]$. Since $\tilde{f} \in R[V]$ and every $I(g), S(g)$ is invertible in $(B \cdot P)_{h}$ we get $\varphi(f) \in(B \cdot P)_{h}$. This shows that $S_{h}=(B \cdot P)_{h}$.

Claim 3. $S_{h}$ is a differentially finitely presented $R$-algebra and $B_{h}$ is a finitely presented $R$-algebra.

Proof. First we prove that $S_{h}$ is differentially finitely presented over $R$. The differential homomorphism $R\{Y\} \rightarrow S \hookrightarrow S_{h}$ maps $H(G)$ onto a unit in $S_{h}$, hence $\varphi$ can be extended to a surjective differential homomorphism $\psi: R\{Y\}\left[H(G)^{-1}\right] \rightarrow S_{h}$ mapping $H(G)^{-1}$ to $h^{-1}$. Since $R\{Y\}\left[H(G)^{-1}\right]$ is a differentially finitely generated $R$-algebra (with generators $\left.Y_{1}, \ldots, Y_{N}, H(G)^{-1}\right)$ it is enough to prove that $\operatorname{Ker} \psi$ is generated by $G$ as a differential ideal. As $\psi$ extends $\varphi$ we have $G \subseteq \operatorname{Ker} \psi$. Conversely if $f \in R\{Y\}$ and $d \in \mathbb{N}$ with $\psi\left(f / H(G)^{d}\right)=0$ we get $f \in \mathfrak{p}$ from $h \neq 0$, hence $H(G)^{n} \cdot f \in[G]$ for some $n \in \mathbb{N}$ by Corollary 6 . This shows that $f / H(G)^{d}$ is in the differential ideal generated by $G$ in $R\{Y\}\left[H(G)^{-1}\right]$. 
Now we show that $B_{h}$ is a finitely presented $R$-algebra. Similar as above we get a surjective $R$-algebra homomorphism $\psi: R\left[V_{B}\right]_{H(G)} \rightarrow B_{h}$ extending $\left.\varphi\right|_{R\left[V_{B}\right]}$ with $\psi H(G)^{-1}=h^{-1}$ and it is enough to show that the ideal $\operatorname{Ker} \psi$ is generated by $G$. If $f \in R\left[V_{B}\right]$ and $d \in \mathbb{N}$ with $\psi\left(f / H(G)^{d}\right)=0$ we get $f \in \mathfrak{p}$. Since $f$ is weakly reduced with respect to $G$ we get $H(G)^{n} \cdot f \in(G)$ for some $n \in \mathbb{N}$ from Corollary 6. Since $G, f$ and $H(G)$ are in $R\left[V_{B}\right], f$ is in the ideal generated by $G$ in $R\left[V_{B}\right]_{H(G)}$. This finishes the proof of claim 3 .

Claims 2 and 3 prove assertions (a) and (b) of Theorem 1.

Claim 4. If $b_{1}, \ldots, b_{m} \in B$ are linearly dependent over $P$, then they are linearly dependent over $R$.

Proof. Take $f_{i} \in R\left[V_{B}\right]$ with $\varphi f_{i}=b_{i}$ and $p_{i} \in R\left[V \backslash V_{B}\right]$, not all contained in $\mathfrak{p}$ with $q:=p_{1} f_{1}+\ldots+p_{m} f_{m} \in \mathfrak{p}$. We may assume that $p_{1} \notin \mathfrak{p}$. Since $q \in R[V], q$ is weakly reduced with respect to $G$. By Corollary 6 there is some $n \in \mathbb{N}$ and polynomials $h_{g} \in R\{Y\}(g \in G)$ such that $H(G)^{n} \cdot q=\sum_{g \in G} h_{g} \cdot g$. Since $H(G), q \in R[V]$ and $G \subseteq R[V]$ we may assume that each $h_{g} \in R[V]$ as well. Since $p_{1} \neq 0$ there is an $R$-algebra homomorphism $\psi: R\left[V \backslash V_{B}\right] \rightarrow R$ with $\psi\left(p_{1}\right) \neq 0$. Clearly $\psi$ can be extended to an $R\left[V_{B}\right]$-algebra homomorphism $\psi: R[V] \rightarrow R\left[V_{B}\right]$. Since all $p_{i}$ are in $R[V]$ we may apply $\psi$ to the equation $H(G)^{n} \cdot\left(p_{1} f_{1}+\ldots+p_{m} f_{m}\right)=\sum_{g \in G} h_{g} \cdot g$. Since $H(G), f_{i} \in R\left[V_{B}\right]$ and $G \subseteq R\left[V_{B}\right]$ we get $H(G)^{n} \cdot\left(\psi\left(p_{1}\right) f_{1}+\ldots+\psi\left(p_{m}\right) f_{m}\right) \in \sum_{g \in G} R\left[V_{B}\right] \cdot g$. Applying $\varphi$ to this equation yields $h^{n} \cdot\left(\varphi\left(\psi\left(p_{1}\right)\right) b_{1}+\ldots+\varphi\left(\psi\left(p_{m}\right)\right) b_{m}\right)=0$. Since $h \neq 0$ and $\varphi\left(\psi\left(p_{1}\right)\right)=\psi\left(p_{1}\right) \neq 0$ the latter equation shows that $b_{1}, \ldots, b_{m}$ are linearly dependent over $R$ and claim 4 is proved.

Claim 4 implies item (c) of Theorem 1 as follows. Suppose $B \otimes_{R} P \rightarrow B \cdot P$ is not injective. Take a minimal $m \in \mathbb{N}$ such that there are $b_{1}, \ldots, b_{m} \in B$ and $p_{1}, \ldots, p_{m} \in P$ with $p_{1} b_{1}+\ldots+p_{m} b_{m}=0$ and $x:=p_{1} \otimes b_{1}+\ldots+p_{m} \otimes b_{m} \neq 0$. Then $b_{1}, \ldots, b_{m}$ are linearly dependent over $P$. So by claim 4 there are $r_{1}, \ldots, r_{m} \in R$ not all zero with $r_{1} b_{1}+\ldots+r_{m} b_{m}=0$. Say $r_{1} \neq 0$. Then $m>1$ and $r_{1} \cdot x=\left(r_{1} p_{2}-r_{2} p_{1}\right) \otimes b_{2}+\ldots+$ $\left(r_{1} p_{m}-r_{m} p_{1}\right) \otimes b_{m}$. From the minimal choice of $m$ we get $r_{1} \cdot x=0$. Let $F$ be the quotient field of $R$. Then $1 \otimes x=\frac{1}{r_{1}} \otimes r_{1} x=0$ in $F \otimes_{R}\left(B \otimes_{R} P\right)$. By claim $1, P$ is a polynomial ring over $R$, hence a flat $R$-algebra. As $B \rightarrow F \otimes_{R} B$ is injective, it follows that $B \otimes_{R} P \rightarrow F \otimes_{R} B \otimes_{R} P$ is injective. So $1 \otimes x=0$ in $F \otimes_{R} B \otimes_{R} P$ implies $x=0$, a contradiction.

Finally we show that $P \cong R\left[V \backslash V_{B}\right]$ can be decomposed as claimed in (d). Let $\rho \in \mathbb{N}$ be strictly bigger than every $\operatorname{ord}_{i} u_{g}(1 \leq i \leq K, g \in G)$. Here $\operatorname{ord}_{i}\left(\partial_{1}^{k_{1}} \ldots \partial_{K}^{k_{K}} Y_{j}\right):=k_{i}$. Let $V_{\emptyset}:=\left\{y \in V \backslash V_{B} \mid \operatorname{ord}_{i} y<\rho(1 \leq i \leq K)\right\}$ and let

$$
\begin{aligned}
& W:=\left\{y \in V \mid \operatorname{ord}_{i} y \leq \rho(1 \leq i \leq K)\right. \text { and } \\
& \left.\operatorname{ord}_{i} y=\rho \text { for at least one } i \in\{1, \ldots, K\}\right\} .
\end{aligned}
$$

For every nonempty subset $\Delta$ of $\left\{\partial_{1}, \ldots, \partial_{K}\right\}$ let

$$
\begin{gathered}
W_{\Delta}:=\left\{w \in W \mid \operatorname{ord}_{i} w=\rho \Longleftrightarrow \partial_{i} \in \Delta(1 \leq i \leq K)\right\} \text { and } \\
V_{\Delta}:=\left\{\partial_{1}^{k_{1}} \ldots \partial_{K}^{k_{K}} w \mid w \in W_{\Delta} \text { and } k_{i}=0 \text { for all } i \in\{1, \ldots, K\} \text { with } \partial_{i} \notin \Delta\right\}
\end{gathered}
$$


So, if $W_{\Delta}=\emptyset$ then $V_{\Delta}=\emptyset$. Also, $y \in V_{\Delta}$ if and only if $y=D w$ for a higher derivative $D$ in the derivatives from $\Delta$.

Claim 5. We have

(i) If $y \in V_{\Delta}$ and $\partial_{i} \in \Delta$, then $\partial_{i} y \in V_{\Delta}$. It follows that $R\left[V_{\Delta}\right]$ together with the derivatives from $\Delta$ is the differential polynomial ring in these derivatives, in the variables from $W_{\Delta}$.

(ii) $V \backslash V_{B}$ is the disjoint union of the $V_{\Delta}(\Delta \subseteq\{1, \ldots, K\})$.

Proof. (i). We have to show $\partial_{i} y \in V$ whenever $y \in V_{\Delta}$ and $\partial_{i} \in \Delta$. Since $y=D w$ for some $w \in W_{\Delta} \subseteq V$ and only derivatives from $\Delta$ appear in $D, y$ cannot be a derivative of any $u_{g}$.

(ii). Clearly $V_{\Delta} \cap V_{\tilde{\Delta}}=\emptyset$, whenever $\Delta \neq \tilde{\Delta}$. Let $y \in V \backslash\left(V_{B} \cup V_{\emptyset}\right)$ be a derivative of $Y_{j}$, hence $\operatorname{ord}_{i} y \geq \rho$ for some $i \in\{1, \ldots, K\}$. Let $\Delta:=\left\{\partial_{i} \mid \operatorname{ord}_{i} y \geq \rho\right\}$ and let $k_{i}:=\min \left\{\operatorname{ord}_{i} y, \rho\right\}(1 \leq i \leq K)$. Then $w:=\partial_{1}^{k_{1}} \ldots \partial_{K}^{k_{K}} Y_{j} \in W_{\Delta}$ and $y \in V_{\Delta}$. This proves (ii).

We define $P_{\Delta}:=\varphi\left(R\left[V_{\Delta}\right]\right)$. By claim 1 and (c) we get (d) from (i) and (ii).

\section{References}

[1] T. Grill, M. Knebusch and M. Tressl, An existence theorem for systems of implicit differential equations, this volume.

[2] E. R. Kolchin, Differential Algebra and Algebraic Groups, Pure Appl. Math. 54, Academic Press, 1973. 